\title{
Air trapping on computed tomography: regional versus diffuse
}

\author{
Firdaus A. Mohamed Hoesein and Pim A. de Jong \\ Affiliation: Dept of Radiology, University Medical Center Utrecht, Utrecht, The Netherlands. \\ Correspondence: Firdaus A. Mohamed Hoesein, University Medical Center Utrecht, Department of Radiology, \\ Heidelberglaan 100, P0 box 855000, 3508 GA, Utrecht, The Netherlands. E-mail: fmohamedhoeseinagmail.com
}

@ERSpublications

Future studies on the distribution of air trapping in the lungs of COPD patients are needed http://ow.ly/86hc304JiIK

Cite this article as: Mohamed Hoesein FA, de Jong PA. Air trapping on computed tomography: regional versus diffuse. Eur Respir J 2017; 49: 1601791 [https://doi.org/10.1183/13993003.01791-2016].

Chronic obstructive pulmonary disease (COPD) is diagnosed and classified by spirometry, assessing the presence of airflow obstruction and extent of forced expiratory flow deterioration. Spirometry, however, is unsuitable for characterising and quantifying the underlying pulmonary pathology of COPD, including alveolar destruction (emphysema) and airway remodelling (large- and small-airway disease). Accurate diagnosis of the pulmonary pathologies underlying COPD is seen as an important step towards better understanding its biology and "personalised" treatment. Computed tomography (CT) of the lungs provides an excellent opportunity to assess COPD in vivo and quantify its macroscopic pathology [1-3]. Emphysema and airway remodelling can be visually assessed and automatically quantified, and several studies have shown associations with morbidity and mortality [4-7]. In addition, CT can provide information on the spatial distribution of disease.

In this issue of the European Respiratory Journal, KARIMI et al. [8] hypothesise that regional air trapping maybe a new imaging marker, in addition to emphysema, large-airway remodelling and diffuse air trapping.

The small airways, commonly defined as those $<2 \mathrm{~mm}$ in diameter, are regarded as the most important site of airflow obstruction in COPD [9]. Measuring small-airway disease on CT can be challenging as these cannot be visualised directly because they are too small and below the resolution of the CT scanner. Indirect measures are thus used to measure small-airway disease on CT. Air trapping on CT, sometimes also referred to as gas trapping, is often used to asses small-airway disease [10]. Pathophysiologically, it is defined as retention of air in the lung distal to an obstruction, but it is difficult to prove this by radiology-pathology correlation studies. To assess air trapping, both inspiratory and some form of expiratory scans are needed.

The definition of regional air trapping on CT consists of three components. First, the trapped areas have a relatively high attenuation on expiratory CT scans. Fully inflated alveoli have an average attenuation of -850 Hounsfield units (HU) and with a good expiratory effort, the average attenuation increases by $150 \mathrm{HU}$. The second component is volume, as areas with air trapping do not lose much of their volume on expiration. The third component is morphology, as air trapping has a sharp delineation at the border of the secondary pulmonary lobule.

The radiological diagnosis of regional air trapping is challenging for several reasons. First, proper expiration is needed for the air trapping to become visible. This needs compliance from patients or research participants, properly trained technicians, and/or spirometric gating. CT technicians tend to train patients to only a limited extent before the CT acquisition and they also sometimes start the scan too

Received: Sept 092016 | Accepted: Sept 212016

Conflict of interest: None declared.

Copyright OERS 2017 
early, before a proper expiration is reached. Second, a very deep expiration tends to make the lung parenchyma, especially in the lower lobes, inhomogeneous. This introduces variation between observers and we often have discussions on the topic of "normal inhomogeneity" during multidisciplinary clinical meetings, especially in military recruits. Furthermore, emphysema or diffuse air trapping may mask regional air trapping and diffuse air trapping is certainly much more difficult to evaluate visually. Finally, it is well known that regional air trapping can be seen in healthy subjects. We previously showed [11] that lobular air trapping was present in $80 \%$ of military recruits, among whom the median number of lobules involved was two with an interquartile range of 1-4. In addition, LEE et al. [12] showed that the frequency and extent of air trapping significantly increased with ageing.

There are several quantitative air trapping measures on CT [13]. A commonly used quantitative measure for air trapping is the ratio between the mean lung density at expiration and inspiration, the so-called $\mathrm{E} / \mathrm{I}$ ratio. Unfortunately, there are no real validated cut-off values for the E/I ratio to differentiate between normal and abnormal, but the higher the E/I ratio, the more air trapping is present. As explained earlier, this can be either due to effort or "real" disease. Another point is that the E/I ratio has not been validated against pathology references.

Using a ratio of the mean lung density to quantify air trapping (i.e. small-airway disease) in COPD patients can be complicated because it needs to be differentiated from air remaining in emphysematous airspaces after exhalation. To solve this issue, GALBÁN et al. [14] introduced a technique called parametric response mapping (PRM), which is able to separate air trapping from air remaining in emphysematous low-attenuation areas. PRM was able to show that COPD subjects with the same forced expiratory volume in $1 \mathrm{~s}$ could show different degrees of air trapping and emphysema, and that in mild-to-moderate COPD, air trapping (termed functional small airway disease in the PRM method) has the greatest importance compared to emphysema [15].

Besides the total extent of disease quantified on chest CT, the distribution of disease is also important and has been shown to be the case in emphysema, for example, to differentiate the upper zone-predominant centrilobular emphysema from the lower zone-predominant pan-lobular emphysema related to $\alpha_{1}$-antitrypsin deficiency. In addition, it has been observed that COPD patients with upper lobe-predominant emphysema have lower pulmonary function and, maybe even more importantly, have a more rapid decrease in pulmonary function [16]. It could be hypothesised that the distribution of small-airway disease, i.e. regional or diffuse, is also of importance in COPD; however, there are no data yet on this topic.

With this in mind, the results of the study by KARIMI et al. [8] are of interest. These authors found that smokers with visually assessed regional air trapping had lower E/I ratios, indicative of less diffuse small-airway disease-related air trapping or better expiratory effort. These smokers also had less emphysema and higher total cell yield in their bronchoalveolar lavage (BAL) fluid but no abnormal distribution of inflammatory cells. Otherwise, the smokers did not differ from nonsmokers or smokers without regional air trapping, or from the COPD patients. The authors concluded that smokers with regional air trapping on CT might be a specific group of smokers who are less susceptible to parenchymal destruction. In the study, the presence of regional air trapping was thus a negative diagnostic sign for COPD in participants with a substantial smoking history.

Several strengths of the study by KARIMI et al. [8] can be identified. The degree of expiration seems good, which is an important condition before air trapping can be assessed. Two experienced readers, who made a consensus reading in case of disagreement, performed the CT reading. The definition of regional air trapping with a minimal threshold of three lobules is a reasonable one and appropriate for this study. The CT study protocol included one expiration coupe per centimetre, while volume scans are the state of the art; however, this is sufficient to assess air trapping in practice. In addition, the extensive additional measures, including BAL, are strengths of this study.

However, there are a few limitations of the study. Regional air trapping was only scored as absent or present (three or more secondary lobules involved) while several studies have shown that some air trapping can also be seen in nonobstructive smokers, as in healthy volunteers [17-19]. This is also reflected in the high and similar prevalence rates of regional air trapping in nonobstructive smokers and healthy volunteers in their study. In addition, the amount of expiration can be a confounder, as good expiration may make air trapping better visible, but it may also reveal some inhomogeneity that may be normal. In a way, the results of KARIMI et al. [8] underscore that the regional air trapping was probably normal, as those with regional air trapping had less emphysema, and were less obstructed and very comparable to the controls. Interestingly, there were no significant differences in residual volumes or total lung capacity, which may serve as a measure of air trapping, between the smokers with and without regional air trapping, which is spurious. Lastly, the E/I ratio, which the authors used to quantify diffuse air trapping, cannot reliably distinguish between air trapping and emphysema. This is also reflected by higher degrees of emphysema in those with higher E/I ratios. 
Despite these limitations, the study sheds new light on the question of whether there is a difference between regional (patchy) air trapping and diffuse air trapping. Because CT enables us to obtain information on the spatial distribution of air trapping, it could provide us with unique information compared to any pulmonary function test. Radiologists are used to qualifying air trapping as present or absent and to grading the severity of air trapping (as mild, moderate or severe), but are generally not used to reporting air trapping in terms of regional versus diffuse forms of air trapping. In addition, visual estimation of the distribution of air trapping can be challenging.

An automatic method to quantify the distribution of air trapping might offer a solution. Recently, HoFF et al. [20] showed that it is possible to provide an automatically derived morphological index of spatial heterogeneity of air trapping in nonemphysematous areas. They showed in two Global Initiative for Chronic Obstructive Lung Disease grade 2 COPD patients that there can be, in addition to severity, a significant difference in spatial distribution of air trapping: one had diffuse air trapping while the other had coalesced air trapping.

Future studies on the distribution of air trapping are needed. These future studies should preferably not use dichotomous visual scores or the E/I ratio to quantify air trapping in COPD, as these methods do not separate it from low-attenuation areas caused by emphysema. It should also be realised that with more advanced measures such as PRM, there is a normal range of air trapping in healthy subjects [21].

\section{References}

1 Camp PG, Ramirez-Venegas A, Sansores RH, et al. COPD phenotypes in biomass smoke-versus tobacco smoke-exposed Mexican women. Eur Respir J 2014; 43: 725-734.

2 Bommart S, Marin G, Bourdin A, et al. Computed tomography quantification of airway remodelling in normal ageing subjects: a cross-sectional study. Eur Respir J 2015; 45: 1167-1170.

3 Mohamed Hoesein FA, de Jong PA. Landmark papers in respiratory medicine: Automatic quantification of emphysema and airways disease on computed tomography. Breathe (Sheff) 2016; 12: 79-81.

4 Mohamed Hoesein FA, de Jong PA, et al. Airway wall thickness associated with forced expiratory volume in 1 second decline and development of airflow limitation. Eur Respir J 2015; 45: 644-651.

5 Oelsner EC, Carr JJ, Enright PL, et al. Per cent emphysema is associated with respiratory and lung cancer mortality in the general population: a cohort study. Thorax 2016; 71: 624-632.

6 Jairam PM, van der Graaf Y, Lammers JW, et al. Incidental findings on chest CT imaging are associated with increased COPD exacerbations and mortality. Thorax 2015; 70: 725-731.

7 Johannessen A, Skorge TD, Bottai M, et al. Mortality by level of emphysema and airway wall thickness. Am J Respir Crit Care Med 2013; 187: 602-608.

8 Karimi R, Tornling G, Forsslund $\mathrm{H}$, et al. Differences in regional air trapping in current smokers with normal spirometry. Eur Respir J 2017; 49: 1600345.

9 Hogg JC, Macklem PT, Thurlbeck WM. Site and nature of airway obstruction in chronic obstructive lung disease. N Engl J Med 1968; 278: 1355-1360.

10 Hansell DM, Bankier AA, MacMahon $\mathrm{H}$, et al. Fleischner Society: glossary of terms for thoracic imaging. Radiology 2008; 246: 697-722.

11 Mets OM, van Hulst RA, Jacobs C, et al. Normal range of emphysema and air trapping on CT in young men. AJR Am J Roentgenol 2012; 199: 336-340.

12 Lee KW, Chung SY, Yang I, et al. Correlation of aging and smoking with air trapping at thin-section CT of the lung in asymptomatic subjects. Radiology 2000; 214: 813-836.

13 Mets OM, Zanen P, Lammers JW, et al. Early identification of small airways disease on lung cancer screening CT: comparison of current air trapping measures. Lung 2012; 190: 629-633.

14 Galbán CJ, Han MK, Boes JL, et al. Computed tomography-based biomarker provides unique signature for diagnosis of COPD phenotypes and disease progression. Nat Med 2012; 18: 1711-1715.

15 Bhatt SP, Soler X, Wang X, et al. Association between functional small airway disease and $\mathrm{FEV}_{1}$ decline in chronic obstructive pulmonary disease. Am J Respir Crit Care Med 2016; 194: 178-184.

16 Mohamed Hoesein FA, van Rikxoort E, et al. Computed tomography-quantified emphysema distribution is associated with lung function decline. Eur Respir J 2012; 40: 844-850.

17 Hashimoto M, Tate E, Watarai J, et al. Air trapping on computed tomography images of healthy individuals: effects of respiration and body mass index. Clin Radiol 2006; 61: 883-887.

18 Mastora I, Remy-Jardin M, Sobaszek A, et al. Thin-section CT finding in 250 volunteers: assessment of the relationship of CT findings with smoking history and pulmonary function test results. Radiology 2001; 218: 695-702.

19 Tanaka N, Matsumoto T, Miura G, et al. Air trapping at CT: high prevalence in asymptomatic subjects with normal pulmonary function. Radiology 2003; 227: 776-785.

20 Hoff BA, Pompe E, Postma DS, et al. Morphological features of non-emphysematous obstruction in COPD. Am J Respir Crit Care Med 2016; 193: Suppl., A5205.

21 Silva M, Nemec SF, Dufresne V, et al. Normal spectrum of pulmonary parametric response map to differentiate lung collapsibility: distribution of densitometric classifications in healthy adult volunteers. Eur Radiol 2016; 26 : 3063-3070. 